\title{
Synaptic Coding of Time-Varying Spike Trains
}

\author{
Michael Stiber, Li Yan, and José P. Segundo ${ }^{\dagger}$ \\ Technical Report HKUST-CS94-10 \\ June 1994
}

\author{
*Department of Computer Science \\ The Hong Kong University of Science and Technology \\ Clear Water Bay, Kowloon, Hong Kong \\ Department of Anatomy and Cell Biology \\ and Brain Research Institute \\ University of California \\ Los Angeles, California 90024, USA
}

¥This work was supported by the Hong Kong Research Grants Council (DAG92/93.EG18, CERG HKUST187/93E) and Trent H. Wells, Jr. Inc. 


\begin{abstract}
Spike-producing neurons produce complex responses to stationary input trains. These responses have been described using techniques from the field of nonlinear dynamics, and are typical of those from periodically perturbed nonlinear oscillators.

Here we are concerned with the effects of nonstationary input trains. We present recent simulation results, largely in agreement with experimental results on a living preparation, emphasizing the relationships between stationary and nonstationary behaviors. The implications for synaptic coding are considered. We suggest that the viewpoint of a neuron as a nonlinear dynamical system has important contributions to make to our understanding of neural computation.
\end{abstract}




\section{Introduction}

Spike-producing neurons produce complex responses to stationary input trains $[1,2,3]$. These responses, previously obscure, have been described using techniques from the field of nonlinear dynamics $[4,5,6,7]$. They are typical of those from periodically perturbed nonlinear oscillators $[8,9,10,11,12,13,14,15,16]$.

Here we are concerned with the effects of nonstationary input trains. We present recent simulation results, largely in agreement with experimental results on a living preparation, and emphasize the relationships between stationary and nonstationary behaviors. Its implications for synaptic coding, the relationship between presynaptic and postsynaptic trains of a neuron [17], are considered. As the synapse is the functional unit of neural networks, its code is the computational unit, modified by connectivity and other factors $[18,19]$.

\section{Methods}

The simulation results were produced using a physiological model of the crayfish slowly adapting stretch receptor organ (SAO), which includes a prototypical inhibitory synapse $[20,6,21]$. This model, like the living preparation, produces periodic, pacemaker outputs when unperturbed, with all interspike intervals equal to $N$, the natural interval.

We sought to duplicate experiments conducted on the SAO, which used frequency-modulated input trains $[22,23,24]$. Presynaptic trains were produced by starting with a modulation, or rate control, waveform $r(t)$, here a triangle wave. This described the instantaneous rate of the presynaptic train, with minimum $1 / I_{m}$, maximum $1 / I_{M}$, and period $P$. Given a presynaptic spike 
at time $s_{0}$, subsequent spike times can be computed from $s_{k+1}=s_{k}+1 / r\left(s_{k}\right)$. The spike train was applied to the simulated neuron as natural IPSPs, and the resultant postsynaptic spikes were assimilated to a point process [25]. Three sets of event times were recorded: the presynaptic times $\left\langle\ldots, s_{k-1}, s_{k}, s_{k+1}, \ldots\right\rangle$, the postsynaptic times $\left\langle\ldots, t_{i-1}, t_{i}, t_{i+1}, \ldots\right\rangle$, and the start time of each cycle of $r(t),\left\langle\ldots, c_{j-1}, c_{j}, c_{j+1}, \ldots\right\rangle$.

Analysis was based on intervals and cross intervals computed from these event times. Intervals were computed for presynaptic and postsynaptic events; $I_{k}=s_{k}-s_{k-1}$ and $T_{i}=t_{i}-t_{i-1}$, respectively. The presynaptic interval was often normalized by the natural interval as $N / I_{k}$. Cross intervals were computed from a postsynaptic event back to the most recent preceding event of another type. One type was $\phi_{i}=t_{i}-s_{k}$, or the phase, and the other was $\psi_{i}=t_{i}-c_{j}$, the modulation phase.

For brevity's sake, only one type of graph is shown for each of the stationary and nonstationary cases; both based on the return map. A return map displays the relationship between an interval or cross interval and a subsequent one, plotted as $T_{i}$ versus $T_{i+1}$, for instance (in this case, termed a first-order interval return map).

For stationary input, each choice of $N / I$ will produce a characteristic set of return maps; we draw a three-dimensional plot of those maps as functions of normalized input rate. For modulated input, $\psi_{i}$ is used as the third dimension to produce the 3-D return maps $\left(T_{i}, T_{i+1}, \psi_{i}\right)$ and $\left(\phi_{i}, \phi_{i+1}, \psi_{i}\right)$. As $r(t)$ is periodic, $\psi_{i}$ uniquely determines input rate, so the $\psi$ axis can also be considered a rate axis. This is the basis of our comparison of parametrized stationary return maps (bifurcation diagrams [12]) with 3-D modulation return maps. 


\section{Results}

Figure 1 compares a stationary response $(\mathrm{A}, \mathrm{B})$ with two different modulation simulations $(\mathrm{C}$ through F); intervals left (A, C, E) and phases right (B, D, F). In all cases, the range of rates was $0.3 \leq N / I_{k} \leq 1.3$, with $\psi_{i}=P / 2$ corresponding to $N / I_{k}=1.3$ and $\psi_{i}=\{0, P\}, N / I_{k}=0.3$.

The stationary plots show the division of the rate scale into periodic, locked responses (where 1-D curves occur) and aperiodic, nonlocked responses (where there are dense bands, mostly representing walkthroughs). These features were duplicated faithfully by slow modulation periods (C, D), where the 1-D curves indicate alternation, the more general, nonstationary counterpart of locking. Faster modulation produced more distorted responses (E, F); however, the basic features of the stationary bifurcation diagrams are still evident. The points also form sharp features with little scatter, indicating that each cycle of the modulation produced identical behaviors, validating the unstated assumption underlying this graph.

\section{Discussion}

The implications of these results for neural computation are potentially far-reaching; we shall only touch upon major points, leaving detailed discussion to ongoing work [26]. Nonstationary, changing neural inputs are the rule rather than the exception in animals, reflecting changes in their environment and their reactions [19]. We therefore view these experiments as critical for elucidating the computations implemented by synaptic coding, modeling for instance repetitive motor activities such as swimming or breathing [22].

These results are in agreement with those found for the SAO, demonstrating the profound 

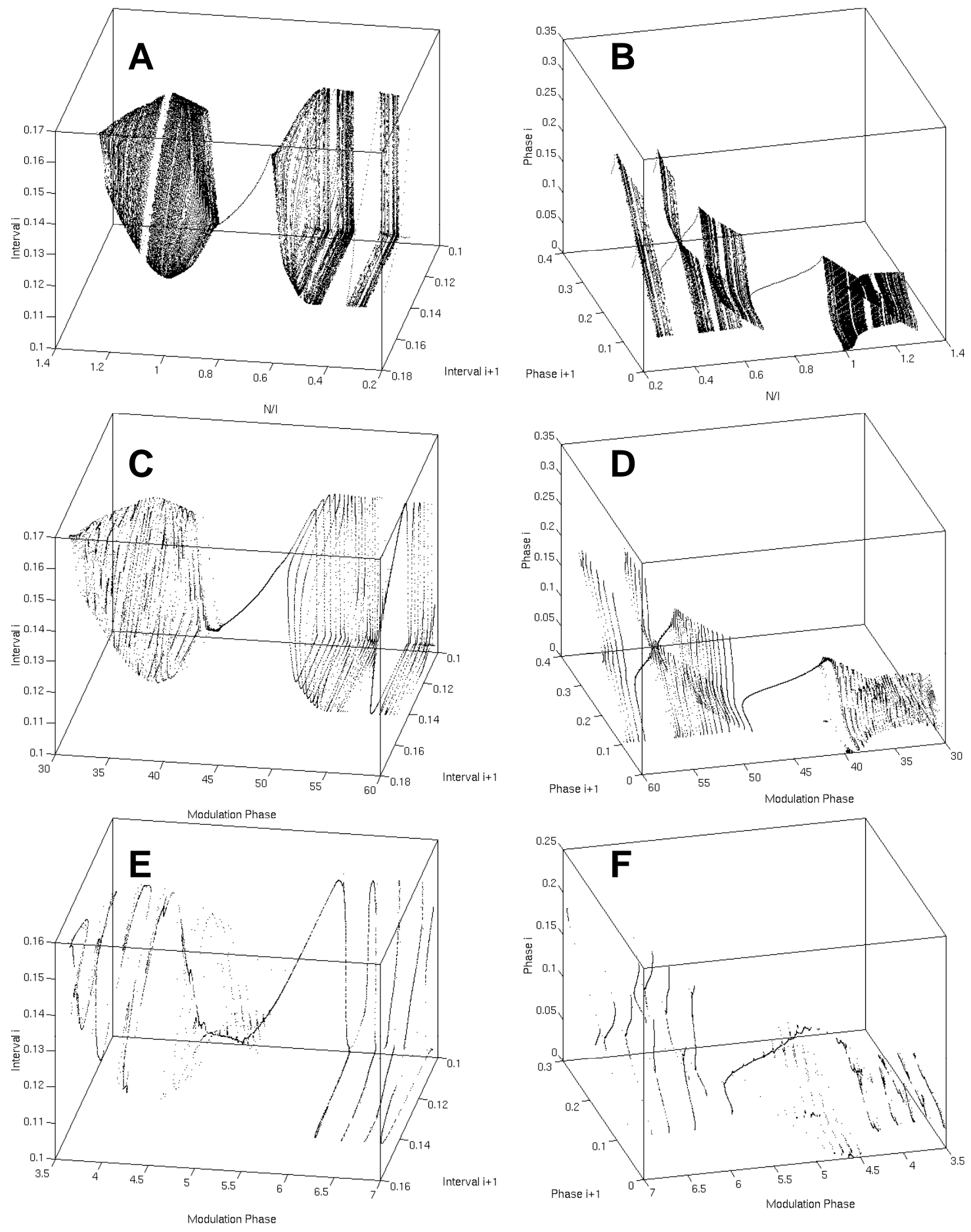

Figure 1: Comparison of stationary and nonstationary responses. Stationary interval (A) and phase (B) return maps, parametrized by normalized input interval $N / I$. Nonstationary 3-D return maps for $P=60 \mathrm{~s}(\mathrm{C}, \mathrm{D})$ and $P=7 \mathrm{~s}(\mathrm{E}, \mathrm{F})$. 
influence stationary behaviors have on neurons' responses to nonstationary input. Additionally, the differences between Figure $1(\mathrm{C}, \mathrm{D})$ and $(\mathrm{E}, \mathrm{F})$ suggest the significance of parameters $P, I_{m}$, and $I_{M}$, as well as average rate slope $\Delta I_{k} / \Delta t$ and range $\left(I_{M}-I_{m}\right)[22,23]$.

Additionally, the synapse's inhibitory nature only shows itself in terms of overall average input and output rates and in the paradoxical accelerating trends during alternating responses. This fact is often ignored in neural network models. We suggest that the viewpoint of a neuron as a nonlinear dynamical system, capable of complex responses to smooth changes in inputs and characterized by dynamical behaviors, bifurcations, spike timing pattern, etc., has important contributions to make to our understanding of neural computation.

\section{References}

[1] D. Perkel, J. Schulman, T. Bullock, G. Moore, and J. Segundo, "Pacemaker neurons: effects of regularly spaced input," Science, vol. 145, pp. 61-3, 1964.

[2] A. Kohn and J. Segundo, "Neuromime and computer simulations of synaptic interactions between pacemakers. Mathematical expansions of existing models," J. Theoret. Neurobiol., vol. 2, pp. 101-25, 1983.

[3] J. Segundo, "Pacemaker synaptic interactions: modeled locking and paradoxical features," Biol. Cybern., vol. 35, pp. 55-62, 1979.

[4] J. P. Segundo, E. Altshuler, M. Stiber, and A. Garfinkel, "Periodic inhibition of living pacemaker neurons: I. Locked, intermittent, messy, and hopping behaviors," Int. J. Bifurcation and Chaos, vol. 1, pp. 549-81, September 1991. 
[5] J. P. Segundo, E. Altshuler, M. Stiber, and A. Garfinkel, "Periodic inhibition of living pacemaker neurons: II. Influences of driver rates and transients and of non-driven postsynaptic rates," Int. J. Bifurcation and Chaos, vol. 1, pp. 873-90, December 1991.

[6] M. Stiber, Dynamics of Synaptic Integration. PhD thesis, University of California, Los Angeles, 1992.

[7] M. Stiber and J. P. Segundo, "Dynamics of synaptic transfer in living and simulated neurons," in IEEE International Conference on Neural Networks, (San Francisco), pp. 75-80, 1993.

[8] C. Hayashi, Nonlinear Oscillations in Physical Systems. Princeton University Press, 1964.

[9] A. Winfree, The Geometry of Biological Time. New York: Springer-Verlag, 1980.

[10] K. Aihara, G. Matsumoto, and Y. Ikegaya, "Periodic and non-periodic responses of a periodically forced Hodgkin-Huxley oscillator,” J. Theor. Biol., vol. 109, pp. 249-69, 1984.

[11] J. Keener and L. Glass, "Global bifurcations of a periodically forced nonlinear oscillator," J. Math. Biol., vol. 21, pp. 175-90, 1984.

[12] P. Bergé, Y. Pomeau, and C. Vidal, Order Within Chaos: A Deterministic Approach to Turbulence. New York: Wiley, 1986.

[13] H. Hayashi and S. Ishizuka, "Chaos in molluscan neuron," in Chaos in Biological Systems (H. Degn, A. Holden, and L. Olsen, eds.), vol. 138 of Series A: Life Sciences, pp. 157-66, New York: Plenum Press, 1987. 
[14] G. Matsumoto, N. Takahashi, and Y. Hanyu, "Chaos, phase locking and bifurcation in normal squid axon," in Chaos in Biological Systems (H. Degn, A. Holden, and L. Olsen, eds.), vol. 138 of Series A: Life Sciences, pp. 143-56, New York: Plenum Press, 1987.

[15] L. Glass and M. Mackey, From Clocks to Chaos. Princeton University Press, 1988.

[16] N. Takahashi, Y. Hanyu, T. Musha, R. Kubo, and G. Matsumoto, "Global bifurcation structure in periodically stimulated giant axons of squid," Physica D, vol. 43, pp. 318-34, 1990.

[17] J. Segundo, M. Stiber, and J.-F. Vibert, "Synaptic coding by spike trains (tutorial)," in IJCNN, (Nagoya, Japan), 25-29 October 1993.

[18] J. Segundo, J.-F. Vibert, M. Stiber, and S. Hanneton, "Synaptic coding of periodically modulated spike trains," in ICNN, (San Francisco), pp. 58-63, 28 March - 1 April 1993.

[19] J. Segundo, M. Stiber, E. Altshuler, and J.-F. Vibert, "Transients in the inhibitory driving of neurons and their post-synaptic consequences," Neuroscience, submitted, 1993.

[20] A. Edman, S. Gestrelius, and W. Grampp, "Analysis of gated membrane currents and mechanisms of firing control in the rapidly adapting lobster stretch receptor neurone," $J$. Physiol., vol. 384, pp. 649-69, 1987.

[21] M. Stiber, J. Segundo, E. Altshuler, and N. Jurisic, "Complex, complex enough, too complex? A comparison of dynamical neural models," Tech. Rep. CS93-11, HKUST Computer Science Department, 1993. 
[22] J. Segundo, J.-F. Vibert, M. Stiber, and S. Hanneton, "Periodically modulated inhibition and its post-synaptic consequences. I. General features. Influences of modulation frequency," Neuroscience, 1994. in press.

[23] J. Segundo, M. Stiber, J.-F. Vibert, and S. Hanneton, "Periodically modulated inhibition and its post-synaptic consequences. II. Influence of pre-synaptic slope, depth, range, noise and of post-synaptic natural discharges," Neuroscience, 1994. in press.

[24] M. Stiber, L. Yan, and J. Segundo, "Synaptic coding of time-varying spike trains," in CNS*94, (Monterey, California), 21-26 July 1994.

[25] D. Cox and V. Isham, Point Processes. London: Chapman and Hall, 1980.

[26] M. Stiber, L. Yan, J. Segundo, J.-F. Vibert, and S. Hanneton, "Periodically modulated inhibition and its postsynaptic consequences. Simulated and living neurons." in preparation, 1994. 